\title{
THE SURFACE MASS DENSITY AND STRUCTURE OF THE OUTER DISK OF NGC 628 *
}

\author{
Stéphane Herbert-Fort ${ }^{1}$, Dennis Zaritsky ${ }^{1}$, \\ Daniel Christlein ${ }^{2}$, Sheila J. Kannappan ${ }^{3}$ \\ ${ }^{1}$ University of Arizona/Steward Observatory, 933 N Cherry Avenue, Tucson, AZ 85721 \\ (email: shf@as.arizona.edu) \\ ${ }^{2}$ Max-Planck-Institut für Astrophysik, Karl-Schwarzschild-Str. 1, 85748 Garching, Germany \\ ${ }^{3}$ University of North Carolina/Department of Physics and Astronomy, 290 Phillips Hall CB 3255, \\ Chapel Hill, NC 27599
}

\begin{abstract}
We study the kinematics of GALEX-selected $\mathrm{H} \alpha$ knots in the outer disk (beyond $R_{25}$ ) of NGC 628 (M74), a galaxy representative of large, undisturbed, extended UV (Type 1 XUV) disks. Our spectroscopic target sample of 235 of the bluest UV knots surrounding NGC 628 yielded $15 \mathrm{H} \alpha$ detections $(6 \%)$, roughly the number expected given the different mean ages of the two populations. The measured vertical velocity dispersion of the $\mathrm{H} \alpha$ knots between $1-1.8 R_{25}(13.5-23.2 \mathrm{kpc})$ is $<11 \mathrm{~km} \mathrm{~s}^{-1}$. We assume that the $\mathrm{H} \alpha$ knots trace an 'intermediate' vertical mass density distribution (between the isothermal $\operatorname{sech}^{2}(z)$ and exponential distributions) with a constant scaleheight across the outer disk $\left(h_{z}=700 \mathrm{pc}\right)$ and estimate a total surface mass density of $7.5 M_{\odot} \mathrm{pc}^{-2}$. This surface mass density can be accounted for by the observed gas and stars in the outer disk (little or no dark matter in the disk is required). The vertical velocity dispersion of the outer disk $\mathrm{H} \alpha$ knots nearly matches that measured from older planetary nebulae near the outskirts of the optical disk by Herrmann et al., suggesting a low level of scattering in the outer disk. A dynamically cold stellar component extending nearly twice as far as the traditional optical disk poses interesting constraints on the accretion history of the galaxy.

Subject headings: galaxies: individual (NGC 628) - galaxies: star clusters - galaxies: structure
\end{abstract}

\section{INTRODUCTION}

The outskirts of a galaxy are expected to host signatures of disk formation and hierarchical accretion because of the long dynamical times at these radii. Toth \& Ostriker (1992) argued that the thinness and coldness of inner galactic disks (inside the optical radius, $R_{25}$ ) posed significant problems for hierarchical models of galaxy formation. Qualitatively, these concerns become more pronounced if one can establish that cold galactic disks extend to even larger radii.

Various studies have revisited the cold disk problem, generally finding that accretion events are less destructive than originally envisioned. Most recently, Kazantzidis et al. (2009) studied the dynamical response of thin galactic disks to bombardment by cold dark matter substructure out to large radii in fully selfconsistent, dissipationless $N$-body simulations. They found that disks survive these bombardments, but that they produce considerable thickening and heating at all radii, substantial flaring, and an increase in the stellar surface density in the disk outskirts (the latter due to outward radial migration of old stars during the growth and redistribution of disk angular momentum). Observations of the dynamical state of outer stellar disks (Christlein \& Zaritsky 2008) demonstrate that outer disks generally continue to obey the flat rotation curves of inner disks, with no increase in the inplane velocity dispersion. Here we present the face-on kinematics of one nearby galaxy, NGC 628 (M74), and

\footnotetext{
* This paper includes data gathered with the 6.5 meter Magellan Telescopes located at Las Campanas Observatory, Chile.
}

compare to both neutral hydrogen and existing stellar measurements at smaller radii. NGC 628 is a prototypical Grand Design spiral galaxy (type SA(s)c, dynamical mass within the studied region $\sim 3.3 \times 10^{11} M_{\odot}$, assuming $v_{\text {rot }}=200 \mathrm{~km} \mathrm{~s}^{-1}$ out to $2.3 R_{25}$; Thilker et al. 2007; Kamphuis \& Briggs 1992), and is by far the largest and most massive member of its small group (the brightest member after NGC 628, UGC1176, is $\sim 4.5$ mag fainter and over $125 \mathrm{kpc}$ away). NGC 628 shows a standard exponential optical light profile to $R_{25}$, with only a very slight possible downbending in the profile to $\sim 1.3 R_{25}$, the extent of the deep optical observations (Natali et al. 1992). Using ultraviolet (UV) imaging, Thilker et al. (2007) classified NGC 628 as a Type 1 extended UV (XUV) disk, due to the structured, UV-bright emission complexes seen in the outer disk. Because the stellar disk appears largely undisturbed (both in optical and UV imaging, and, as we will show, from the kinematics) and because NGC 628 dominates the dynamics of its local environment, we treat it as representative of large, isolated spiral galaxies in the nearby universe.

The outer disks of galaxies have received much recent attention, both observational (Thilker et al. 2007; Gil de Paz et al. 2007; Zaritsky \& Christlein 2007; Christlein \& Zaritsky 2008; Herbert-Fort et al. 2009; Truijllo et al. 2009; Herrmann et al. 2009) and theoretical (Bush et al. 2008; Roškar et al. 2008a,b; Kazantzidis et al. 2009). The surge in interest in outer disks has been fueled by recent ultraviolet (UV) observations of nearby disks with the GALEX satellite (Martin et al. 2005; Thilker et al. 2007). Previously, Ferguson et al. (1998) had used deep $\mathrm{H} \alpha$ imaging to dis- 
cover star formation in an extended component around three nearby galaxies, yet the ubiquity of this component in other disks remained largely unrecognized until the UV observations.

This neglect stemmed in part from the fact that $\mathrm{H} \alpha$ traces a limited subpopulation of the outer disk, namely those regions with $\mathrm{O}$ and $\mathrm{B}$ stars. Even relatively young regions may lack $\mathrm{OB}$ stars because they are older than $10 \mathrm{Myr}$ or because they simply did not form such massive stars (Ferguson et al. 1998; Herbert-Fort et al. 2009; Pflamm-Altenburg \& Kroupa 2008). As such, the ubiquity of outer disk star formation is somewhat concealed. These barriers are removed with UV observations and we now know that many nearby galaxies host young outer disk stellar populations (> 30\%; Thilker et al. 2007; Zaritsky \& Christlein 2007). While galactic interactions can dramatically increase the level of outer disk star formation (see the wellknown cases of M83, NGC 4625 and M94; Thilker et al. 2005; Gil de Paz et al. 2005; Trujillo et al. 2009), even isolated galaxies show low-levels of ongoing star formation in their outer parts (Ferguson et al. 1998; Christlein \& Zaritsky 2008; Herbert-Fort et al. 2009). However, the $\mathrm{H} \alpha$ knots provide the bright emission lines that make it possible to measure the kinematics we present here.

While the gaseous outer disk of NGC 628 has been well-studied, for example by Kamphuis \& Briggs (1992), the stellar component near the outskirts of the disk remains poorly characterized. Observers have acquired deep broadband imaging of the outer disk of NGC 628 (e.g. Natali et al. 1992), however the diffuse light becomes difficult to reliably trace fainter than $\sim 28 \mathrm{mag}$ $\operatorname{arcsec}^{-2}$ in $V$, or beyond $\sim 1.3 R_{25}$. To sidestep the difficulty of obtaining detailed kinematics from low surface brightness emission, Herrmann et al. (2009) use planetary nebulae (PNe) across the face of NGC 628 to trace the underlying stellar distribution and to estimate the total mass density of the disk using the kinematic approach of van der Kruit (1988) (specifically, by measuring the vertical velocity dispersion of a tracer of the disk mass distribution to estimate the underlying surface mass density). In principle, the $\mathrm{PNe}$ provide an excellent approach to the problem, with large numbers of PNe available for analysis. Unfortunately for our purposes, the Herrmann et al. (2009) study of PNe in NGC 628 is mostly constrained to the inner disk (only two of their PNe are beyond $R_{25}$; their study of M83 and especially M94 yield more PNe in those outer disks, however those are interacting systems for which the kinematics are complicated by recent events). The current work represents the first to analyze the disk kinematics out to $1.8 R_{25}$ in NGC 628 . Nevertheless, the results from Herrmann et al. (2009) at the largest radii $\left(\sim R_{25}\right)$ provide an interesting comparison to the results found here, especially because the PNe trace a population of objects that is on average at least $100 \times$ older than the $\mathrm{H} \alpha$ knots considered here.

We use multiobject spectroscopy to search for $\mathrm{H} \alpha$ emission associated with blue GALEX sources in the outer disk of NGC 628. We measure the dispersion of the $\mathrm{H} \alpha$ knot vertical velocity distribution, estimate the surface density of the outer disk, and determine if the baryonic material can account for the inferred mass. We then com- pare the results from the young $\mathrm{H} \alpha$ knots to those from the $\mathrm{PNe}$ and consider the implications on disk evolution from the two measurements. Section 2 presents our sample selection, observations and data reductions. Section 3 presents our analysis, results and discussion, including our measurement of the vertical velocity dispersion of the $\mathrm{H} \alpha$ knots and the surface mass density of the outer disk. Section 4 presents a summary and our conclusions.

\section{SAMPLE SELECTION, OBSERVATIONS, AND DATA REDUCTION}

We selected our target sample from sources identified in GALEX imaging of NGC 628 by Zaritsky \& Christlein (2007). We target 'blue' sources with FUV - NUV $<1$ and NUV $<25$, corresponding to the expected colors of star clusters with ages $<360$ Myr. We observed two fields that cover the outer disk of NGC 628 with the Inamori Magellan Areal Camera and Spectrograph (IMACS; Dressler et al. 2006) using the 'short' f/2 camera mode and the 600 line grating centered at $7695 \AA$, providing a spectral resolution of $\sim 0.6 \AA$ pixel $^{-1}$, or $\sim 27 \mathrm{~km} \mathrm{~s}^{-1}$ pixel $^{-1}$ near $\mathrm{H} \alpha$, on August 2, 3, and 4, 2005 (UTD). The IMACS field of view in the short camera mode is $\sim 27^{\prime}$ on a side, providing coverage out to $3 R_{25}\left(R_{25}=5.2\right.$ arcmin; Kamphuis \& Briggs 1992). The IMACS plate scale in our setup is 0.2 arcsec pixel ${ }^{-1}$. We cut 162 and 151 slits in the masks of the northeastern and southwestern fields, respectively, giving priority to the brightest GALEX sources with the bluest FUV - NUV colors (clusters with $\mathrm{H} \alpha$ emission are expected to have ages $<10 \mathrm{Myr}$, powered by massive stars that will make the clusters blue in the GALEX bands). The seeing remained below $1^{\prime \prime}$ during the observations. We observed the northern field for a total of 18000 seconds, or 5 hours, and the southern field for half that time. To be certain that no significant mask/sky alignment drifts occurred during the overall integrations, we observed neither field for more than 2700 seconds in a single exposure. We observed HeNeAr comparison arc lamps through each mask for wavelength calibration.

We reduce the data with the Carnegie Observatories System for MultiObject Spectroscopy (COSMOS; A. Oemler et al.) package and extract individual 2D rectified, wavelength-calibrated and sky-subtracted spectra in the wavelength range 5600 - $9000 \AA$. Using standard IRAF routines ${ }^{1}$, we median combine the individual skysubtracted spectra corresponding to each knot, rejecting pixel values $>3 \sigma$ from the median of the pixel stack to eliminate cosmic rays.

\section{ANALYSIS, RESULTS \& DISCUSSION}

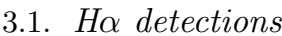

We begin our spectral analysis by visually inspecting all of the sky-subtracted and combined spectra to search for detectable $\mathrm{H} \alpha$ flux from the targeted UVbright knots. For any cases with visually-detected $\mathrm{H} \alpha$ emission, we average the rows showing signal and measure the radial velocity centroid from this final 1D spec-

1 IRAF is distributed by the National Optical Astronomy Observatories, which are operated by the Association of Universities for Research in Astronomy, Inc., under cooperative agreement with the National Science Foundation. 
trum. The procedure is designed such that we may exclude any rows that have minor overlaps with neighboring spectra or contain any remaining artifacts.

We estimate the line centroid measurement errors to be $\sim 8 \mathrm{~km} \mathrm{~s}^{-1}$ from fits to the bright $\mathrm{OH} 6498.72 \AA$ sky line from numerous (pre sky-subtracted) spectra. This measurement error is a lower limit to the $\mathrm{H} \alpha$ line measurement error because the sky line is typically brighter and cleaner than the $\mathrm{H} \alpha$ detections in our sample. We apply a constant $-28.4 \mathrm{~km} \mathrm{~s}^{-1}$ heliocentric velocity correction to the radial velocities of the $\mathrm{H} \alpha$ knots; the difference in the heliocentric correction between consecutive nights is $\sim 0.1 \mathrm{~km} \mathrm{~s}^{-1}$, or $\sim 1 \%$ of the typical line centroid measurement error.

Of the 313 slits on the two IMACS masks, $\sim 25 \%$ returned unusable spectra due to overlapping spectra or other artifacts in the data. We therefore only search for $\mathrm{H} \alpha$ emission in the remaining 235 spectra. Fifteen of these spectra (or $\sim 6 \%$ ) yield detectable $\mathrm{H} \alpha$ emission. Figure 1 presents a few of our final 1D spectra illustrating the range of strength of the $\mathrm{H} \alpha$ detections. Our $6 \%$ return is roughly what is expected given the difference in ages between the 'blue' GALEX-detected knots targeted here $(<360 \mathrm{Myr})$ and $\mathrm{H} \alpha$ knots $(\sim 10 \mathrm{Myr})-$ especially when considering that priority was given to the bluest and brightest GALEX knots (so most targeted GALEX knots are likely $<200 \mathrm{Myr}$ old). We plot the locations of these fifteen knots in Figure 2 and list the relevant information in Table 1. The physical scale of the individual $\mathrm{H} \alpha$ knots (calculated from the extent of $\mathrm{H} \alpha$ emission along the slit) range from 25 to $92 \mathrm{pc}$, with a median value of $67 \mathrm{pc}$ (adopting a distance of $8.6 \mathrm{Mpc}$ to NGC 628 , determined using the planetary nebula luminosity function; Herrmann et al. 2008).

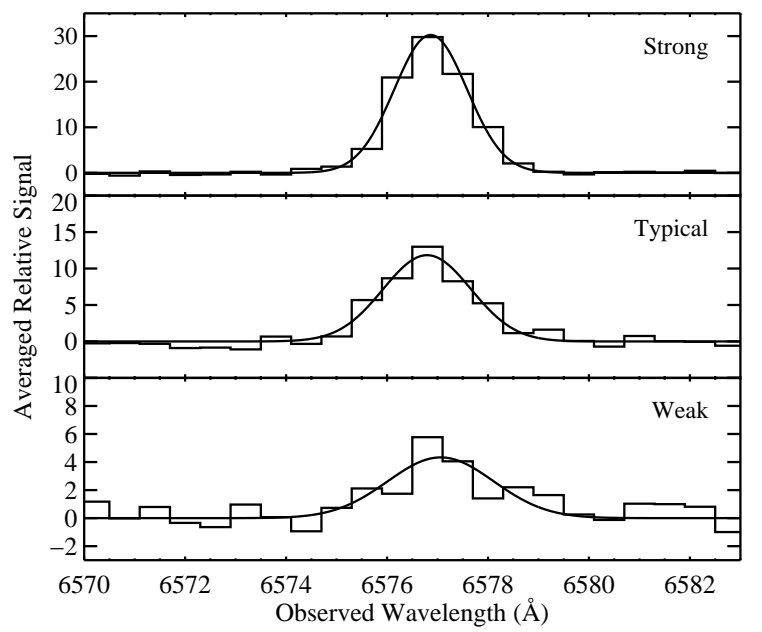

FIG. 1.- Three of our final 1D spectra, illustrating the range of strength of the $\mathrm{H} \alpha$ detections. Note the different $y$-axis ranges. Qualitatively, our final sample is composed of two 'Strong', nine 'Typical', and four 'Weak' $\mathrm{H} \alpha$ detections.

\subsection{Ho knot velocities in the disk frame and $\sigma_{z}$}

Our present aim is to measure the vertical velocity dispersion of the knots in the outer disk of NGC

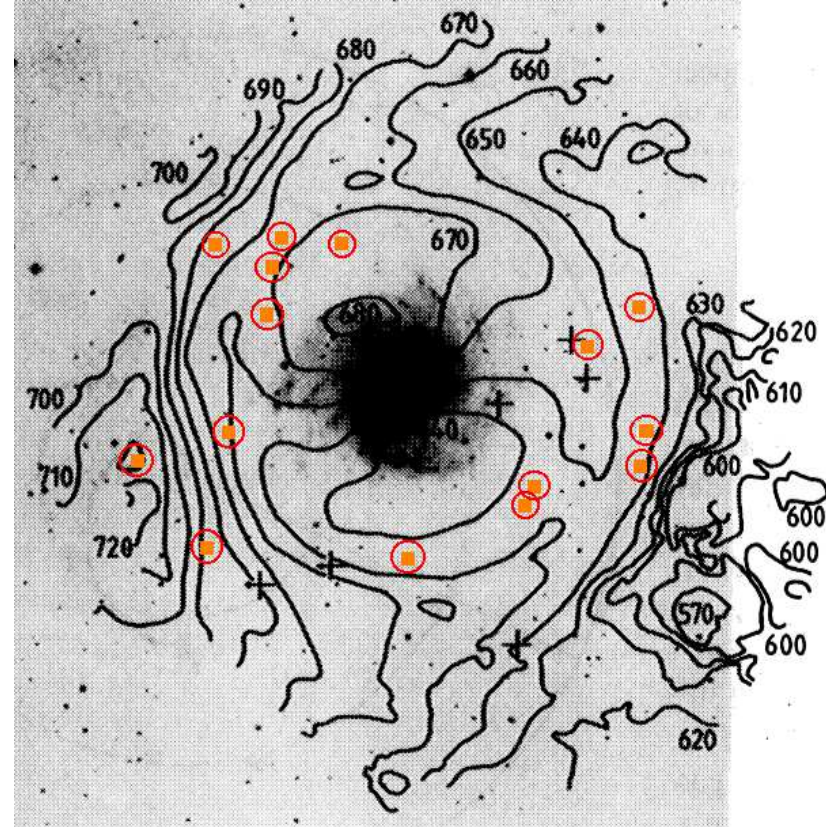

Fig. 2.- Figure 5 of Kamphuis \& Briggs (1992) showing the velocity field of the $\mathrm{H}$ I-emitting gas disk surrounding NGC 628 , with our $15 \mathrm{H} \alpha$ detections overplotted as encircled filled squares. The image is $\sim 30^{\prime}$ on a side. The $\mathrm{H} \alpha$ knots are found between $\sim 1$ and $1.8 R_{25}(13.5-23.2 \mathrm{kpc})$

TABLE 1

$\mathrm{H} \alpha$ DETECTIONS

\begin{tabular}{lccccr}
\hline \hline RA (J2000) & Dec $(\mathrm{J} 2000)$ & $R / R_{25}$ & $v_{\text {helio }}$ & $v_{T H}$ & $\Delta v$ \\
\hline $01: 36: 04.47$ & $+15: 45: 27.3$ & 1.76 & 640 & 641 & -1 \\
$01: 36: 05.40$ & $+15: 44: 23.3$ & 1.76 & 668 & 642 & 26 \\
$01: 36: 06.53$ & $+15: 49: 37.0$ & 1.72 & 652 & 647 & 5 \\
$01: 36: 14.07$ & $+15: 48: 19.0$ & 1.32 & 667 & 652 & 15 \\
$01: 36: 20.94$ & $+15: 43: 33.5$ & 1.17 & 644 & 643 & 1 \\
$01: 36: 22.12$ & $+15: 42: 58.8$ & 1.20 & 628 & 643 & -15 \\
$01: 36: 38.71$ & $+15: 40: 52.1$ & 1.19 & 651 & 642 & 9 \\
$01: 36: 51.02$ & $+15: 52: 03.6$ & 1.06 & 680 & 684 & -4 \\
$01: 36: 59.23$ & $+15: 52: 13.6$ & 1.29 & 657 & 670 & -13 \\
$01: 37: 00.35$ & $+15: 51: 19.1$ & 1.20 & 659 & 675 & -16 \\
$01: 37: 00.94$ & $+15: 49: 49.7$ & 1.04 & 658 & 674 & -16 \\
$01: 37: 05.30$ & $+15: 44: 45.3$ & 1.18 & 639 & 649 & -10 \\
$01: 37: 09.28$ & $+15: 41: 14.0$ & 1.70 & 704 & 679 & 25 \\
$01: 37: 10.06$ & $+15: 52: 08.9$ & 1.64 & 664 & 677 & -13 \\
$01: 37: 17.86$ & $+15: 43: 51.0$ & 1.79 & 656 & 711 & -55
\end{tabular}

* all velocities in $\mathrm{km} \mathrm{s}^{-1}$. $v_{\text {helio }}$ refers to the heliocentric velocities of the $\mathrm{H} \alpha$ knots, while $v_{T H}$ refers to THINGS heliocentric H I-emitting gas velocities measured by Walter et al. (2008). $\Delta v=v_{\text {helio }}-v_{T H}$

$628\left(\sigma_{z}\right.$, with $z$ defined perpendicular to the disk plane). Given the low inclination of NGC $628\left(i \sim 6^{\circ}\right.$; Kamphuis \& Briggs 1992), the observed line-of-sight velocities are almost entirely due to the actual vertical velocities. However, as Kamphuis \& Briggs (1992) have shown, the outer gas disk of NGC 628 is chaotic and non-uniformly rotating. Whether this is also true for the outer stellar disk is unknown, and therefore whether we should use the previous orientation parameters obtained for the inner disk or some other ones is unclear. We therefore present results using various models for the disk orientation and kinematics, and accept the model with the lowest resulting velocity dispersion as the one most likely to be correct. Results of the following analy- 
sis will be discussed further in $\S 4$.

We use a maximum likelihood approach to estimate the Gaussian parameters best describing the unbinned relative velocity $(\Delta v)$ distributions. Specifically, we search a grid of mean and dispersion values (from -50 to 50 $\mathrm{km} \mathrm{s}^{-1}$ and 1 to $100 \mathrm{~km} \mathrm{~s}^{-1}$, respectively) for the combination that yields the highest total probability (likelihood) of the Gaussian function, using the unbinned $\Delta v$ values as the independent variable. Our velocity dispersion estimates will provide only a single value for the radial range spanned by the knots $\left(\sim 1-1.8 R_{25}\right)$. We have insufficient detections to measure the velocity dispersion as a function of radius. The following analysis will therefore consider the outer disk as a constant velocity dispersion, fixed-height component, and the values derived only represent the disk between $\sim 1-1.8 R_{25}$. In support of this approach, note that the simulations of Kazantzidis et al. (2009) predict a roughly constant velocity dispersion in the outer disk.

We examine four models for the outer disk orientation and kinematics. First, we test a purely face-on disk by assuming a constant $656 \mathrm{~km} \mathrm{~s}^{-1}$ systemic velocity. This 'model' yields a velocity dispersion of $18 \pm 5 \mathrm{~km} \mathrm{~s}^{-1}$. Second, we adopt the parameters of the inner neutral hydrogen gas disk of Kamphuis \& Briggs (1992) (inclination $i=6.5^{\circ}$, major axis position angle $P A=25^{\circ}$ and a mean velocity of $656 \mathrm{~km} \mathrm{~s}^{-1}$ ) and a rotation velocity of $170 \mathrm{~km} \mathrm{~s}^{-1}$ (Fathi et al. 2007) and find a velocity dispersion of $19 \pm 5 \mathrm{~km} \mathrm{~s}^{-1}$. Third, we adopt the two-component model of Kamphuis \& Briggs (1992) $\left(i=13.5^{\circ}\right.$ and $P A=75^{\circ}$ beyond $1.2 R_{25}$, using the same inner disk values as the previous case for the knots found at $R<1.2 R_{25}$ ) and find a velocity dispersion of $31 \pm 10$ $\mathrm{km} \mathrm{s}^{-1}$. Finally, we adopt the neutral gas velocities from The H I Nearby Galaxy Survey (THINGS; Walter et al. 2008) at the position of each knot and find a velocity dispersion of $19 \pm 6 \mathrm{~km} \mathrm{~s}^{-1}$. Figure 2 shows the spatial distribution of the knots around the gas disk of NGC 628 , and THINGS gas velocities at the positions of each knot are listed in Table 1. This 'model' tests for the possibility that the knots have low velocity dispersion around a complex velocity field defined by the $\mathrm{H}$ I-emitting gas. Given the result that the velocity dispersion is similar to the simple disk models, we conclude that there is no evidence for highly complex kinematics.

The velocity dispersion estimates generally cluster around $20 \mathrm{~km} \mathrm{~s}^{-1}$ and agree to within $1 \sigma$ (although the one estimate at $31 \pm 10 \mathrm{~km} \mathrm{~s}^{-1}$ is just consistent with the others). The general agreement, independent of the model used, suggests that the dominant source of uncertainty is currently the statistical one associated with the limited sample size. Because the fourth 'model' is taken directly from the $\mathrm{H}$ I data and produces a velocity dispersion as tight as do the other models, we adopt this model for the remainder of the paper.

Figure 3 shows the distribution of velocities relative to the Walter et al. (2008) gas disk values (the fourth model), binned by $15 \mathrm{~km} \mathrm{~s}^{-1}$. We removed a slight, but significant, mean velocity offset $\left(17 \mathrm{~km} \mathrm{~s}^{-1}\right)$ when evaluating the velocity dispersion; Table 1 and Figure 3 include this correction. We attribute this shift to a velocity zero point difference in the various datasets although we were unable to directly identify the cause (Figure 2 shows that the $\mathrm{H} \alpha$ knots are well-distributed across the face of the disk; we therefore do not expect that the offset is due to an undersampling of the relative velocity distribution). When we exclude the knot at 01:37:17.86, $+15: 43: 51.0$ with $\Delta v=v_{\text {helio }}-v_{T H}=-55 \mathrm{~km} \mathrm{~s}^{-1}$, which lies on the high velocity cloud (HVC) described in Kamphuis \& Briggs (1992), we arrive at our best (lowest and most likely) estimate of the observed $\mathrm{H} \alpha$ knot velocity dispersion, $14 \pm 4 \mathrm{~km} \mathrm{~s}^{-1}$. Excluding the same knot from the other models does not significantly change their values. The Gaussian representing this final unbinned $\Delta v$ distribution is overplotted in Figure 3 (for presentation, the height of the Gaussian is determined by a fit to the binned $\Delta v$ distribution; the dispersion is the only value used in our analysis, however).

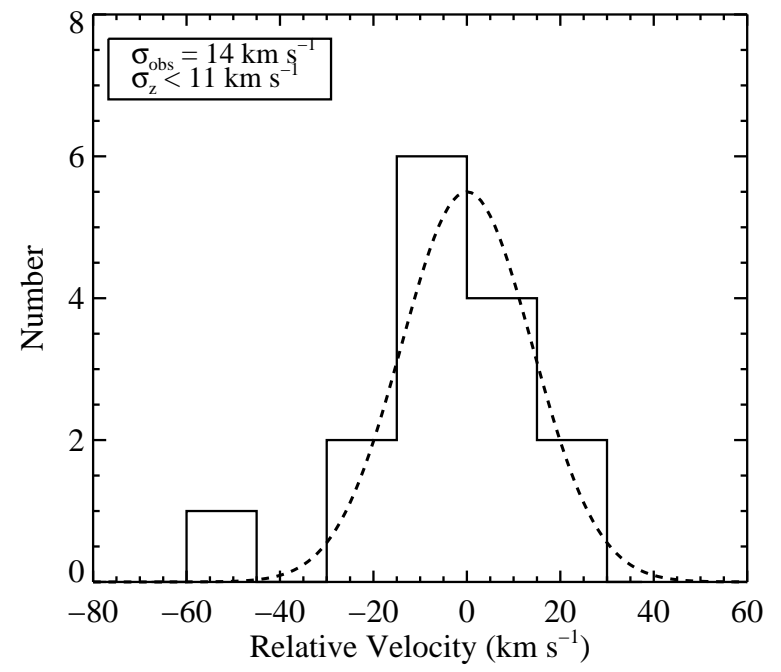

FIG. 3.- Distribution of $\mathrm{H} \alpha$ knot vertical velocities, relative to THINGS (Walter et al. 2008) neutral gas measurements. The outlier is the source detected on the high velocity cloud; we ignore this source in the analysis because its relation to the gas disk is uncertain.

We now consider the degree to which our observational errors inflate the velocity dispersion measurement $\left(\sigma_{o b s}=14 \mathrm{~km} \mathrm{~s}^{-1}\right)$. Subtracting our previous velocity error estimate $\left(8 \mathrm{~km} \mathrm{~s}^{-1}\right)$ in quadrature from the measured velocity dispersion, we estimate that $\sigma_{z}<11 \mathrm{~km} \mathrm{~s}^{-1}$. We emphasize that $\sigma_{z}$ is likely to be an upper limit to the vertical velocity dispersion because 1) the measurement uncertainty is larger than $\left.8 \mathrm{~km} \mathrm{~s}^{-1}, 2\right)$ the uncertainty in the reference $\mathrm{H}$ I-emitting gas velocities was ignored, and 3) we may have missed the correct model for the underlying disk kinematics.

Our low velocity dispersion measurement suggests a relatively undisturbed stellar disk, which at first seems to contrast with the Kamphuis \& Briggs (1992) observation of a chaotic outer gas disk. The Kamphuis \& Briggs (1992) observation was strongly influenced by the HVCs and kinematic features at the largest radii, however-the bulk of the gas disk is in fact rather undisturbed (see Figure 2). Nevertheless, a difference between a relatively undisturbed stellar disk and a disturbed gas disk might be expected, considering the results of Thilker et al. (2007) and Moffett et al. (2009), who find that Type 
1 XUV disks (of which NGC 628 is a member) cover a wide range of galaxy masses, colors and morphologies, and that these disks may in fact be associated with minor interactions or accretion. The disturbed structure observed in the gas may result from an interaction event (Kamphuis \& Briggs 1992) to which the stellar disk was less sensitive. Such an event may have then triggered the Type 1 XUV disk that is observed today.

\subsection{Outer disk scale height $h_{z}$}

Assuming that the $\mathrm{H} \alpha$ knot density has the same vertical distribution as the underlying mass density distribution of the disk, for an adopted scaleheight $h_{z}$ we constrain the surface mass density of the outer disk $\Sigma$ using our measurement of $\sigma_{z}$. We will return to discuss the validity of this assumption, although we do not have any alternative given our current data. The relevant equation (van der Kruit 1988) is

$$
\sigma_{z}^{2}=K G \Sigma h_{z}
$$

where $K=1.7051 \pi$ for an 'intermediate' vertical mass density distribution (between the isothermal $\operatorname{sech}^{2}(z)$ and exponential distributions) and $G$ is the gravitational constant. The relation was constructed by solving the Boltzmann and Poisson equations for plane parallel layers. For a galaxy with a flat rotation curve, the plane parallel case provides a realistic model from which to estimate the surface mass density (van der Kruit 1988). Because Christlein \& Zaritsky (2008) found mostly flat rotation curves in a sample of 17 nearby outer disks, we expect the plane parallel framework to remain valid in the outer regions (we further assume a constant scale height over the radial range of the knots). Corrections to the relation from a dark halo term are negligibly small at low $z$ van der Kruit 1988); however, in the outer disk the baryonic disk contribution to the mass budget presumably drops more rapidly with radius than does the dark matter contribution, and so the dark matter may in fact not be negligible in the outer disk. In the following section we show that the van der Kruit (1988) relation is indeed appropriate at these radii (we find that no dark matter in the disk is required to explain existing observations, even when assuming a rather large value for $h_{z}$ ).

Because NGC 628 is nearly face-on, we must estimate the scale height $h_{z}$ of the tracer particles (the knots) indirectly. Herrmann et al. (2009) argue, based on stability arguments and the observed velocity dispersion of $\mathrm{PNe}$, that the scaleheight near the edge of the region they probe $(\sim 11 \mathrm{kpc})$ must be $>400 \mathrm{pc}$. The vertical disk flaring suggested by the PNe analysis resembles the known flaring of gas disks in their outer regions, with neutral hydrogen gas scale heights near $\sim 700 \mathrm{pc}$ at the $R / R_{25}$ distances considered here (Merrifield 1992; Corbelli \& Salpeter 1993; Olling 1996). Because the $\mathrm{H} \alpha$ knots are young and because they lie at radii larger than those probed by the PNe, we adopt a scale height for the knots in line with that of the flaring gas, $h_{z}=700 \mathrm{pc}$ at $1.3 R_{25}$, the median radius of our $\mathrm{H} \alpha$ detections. Recall however that we treat the outer disk as a fixed-height component. In reality, $h_{z}$ may range between $\sim 400 \mathrm{pc}$ and $\sim 1000 \mathrm{pc}$ over the radii covered by our detections. Assuming a constant 700 pc over the entire outer disk therefore implies a large uncertainty in the final outer disk mass, which we assume to be $\sim 20 \%$. This level of uncertainty will not significantly affect our conclusions, however. Finally, we note that our assumption that the $\mathrm{H} \alpha$ knots trace the same mass distribution as the outer disk gas is different from what one would expect in the inner disk. In the inner disk, molecular clouds (and their $\mathrm{H} \alpha$ knots) have a smaller vertical scaleheight than the mass, which is dominated by the stellar component. In the outer disk, however, where the dominant baryonic component is the gas (as we show below), our assumption is much more appropriate. Whether the $\mathrm{H} \alpha$ knots trace the neutral gas is more uncertain, although where examined in detail the correspondence appears good (Thilker et al. 2005; Herbert-Fort et al. 2009). In view of this, a large scaleheight for the outer stellar disk should be expected. We caution however that if a significant amount of mass extends to heights larger than the 700 pc scaleheight assumed for the knots, we are not sensitive to it with the $\mathrm{H} \alpha$ knot measurement.

\subsection{Outer disk mass density}

We now estimate the mass density of the outer disk in order to determine the relative contributions of stars and gas to the total outer disk mass. Using $\sigma_{z}=11 \mathrm{~km} \mathrm{~s}^{-1}$ and our adopted $h_{z}=700 \mathrm{pc}$, we calculate that the outer disk surface mass density $\Sigma$ is $7.5 \mathrm{M}_{\odot} \mathrm{pc}^{-2}$. The total mass between $1-1.8 R_{25}$ is then $\sim 8.4 \times 10^{9} M_{\odot}$.

We now use results from deep optical imaging of the outer regions of NGC $628\left(\mu_{V} \sim 27 \mathrm{mag} \operatorname{arcsec}^{-2}\right.$ at 1.3 $R_{25}$; Natali et al. 1992) to estimate that the mass-tolight ratio $\Upsilon$ of the outer disk is $\sim 18$. The outer disk mass is therefore not primarily in the form of normal stars. For comparison, Herrmann et al. (2009) found that the PNe velocity dispersions imply $\Upsilon=1.4$ for the inner disk. Reconciling the outer and inner disk $\Upsilon$ values would require a factor of 10 changes in $\sigma_{z}$ or $\Sigma$, neither of which is that poorly constrained. Therefore, as one might expect, we detect a significantly higher $\Upsilon$ in the outer regions. If we adopt $\Upsilon=3$ as an upper limit for an old outer disk stellar population (Bell \& de Jong 2001), we find that the dark material in the outer disk must be at least five times more massive than the stellar component. In order to match the $\mu_{V} \sim 27 \mathrm{mag} \operatorname{arcsec}^{-2}$ observation, an outer disk stellar population with $\Upsilon<3$ would require $\Sigma_{\star}<1.3 M_{\odot} \mathrm{pc}^{-2}$ and $M_{\star}<1.4 \times 10^{9} M_{\odot}$. This leaves $>7 \times 10^{9} M_{\odot}$ of dark material to be accounted for.

We now consider the neutral gas component of the outer disk and whether it can account for the optically non-luminous mass. Using the outer disk H I-emitting gas mass from Kamphuis \& Briggs (1992) (over the relevant radial range), $\sim 6 \times 10^{9} M_{\odot}$, we estimate the total outer disk baryonic mass (atomic gas + stars) to be between $\sim 6 \times 10^{9}-7.4 \times 10^{9} M_{\odot}$. Comparing this total baryonic mass to the total mass required by the $\mathrm{H} \alpha$ knot velocity dispersion $\left(\sim 8.4 \times 10^{9} M_{\odot}\right)$, we find that the baryonic matter (mainly the $\mathrm{H}$ I-emitting gas) can account for nearly all of the measured outer disk mass (note that we have ignored any molecular gas contribution). Our assumption that the dark matter contribution to the disk is negligible at large radii appears justified, as does our claim that the gaseous component dominates the baryon budget. 


\subsection{Star formation history constraints}

A separate intriguing question is whether star formation has occurred at the current rate for the entire lifetime of this galaxy. The result of such a stellar component could, in principle, violate the surface brightness or surface mass density measurements. Independent of the surface brightness measurement we can simply calculate whether the integrated star formation results in a mass density in conflict with the measurement. Using deep $\mathrm{H} \alpha$ imaging of three nearby late-type spiral galaxies (one of which is the current focus; NGC 628, NGC 1058 and NGC 6946), Ferguson et al. (1998) found their outer disk star formation rate densities to be between $\sim 0.01-0.05 M_{\odot} \mathrm{pc}^{-2} \mathrm{Gyr}^{-1}$. For the following exercise, we adopt a SFR density of $0.03 M_{\odot} \mathrm{pc}^{-2}$ $\mathrm{Gyr}^{-1}$ for the outer disk of NGC 628. For comparison to the Ferguson et al. (1998) SFR densities, Trujillo et al. (2009) used GALEX FUV imaging to measure the SFR density in the outer disk of M94 and found $\sim 0.4 M_{\odot} \mathrm{pc}^{-2}$ $\mathrm{Gyr}^{-1}$. The star formation in the outer regions of M94 is unusually large however, producing an anti-truncated optical disk light profile (NGC 628 shows a standard exponential optical light profile to $R_{25}$, with only a very slight possible downbending in the profile to $\sim 1.3 R_{25}$, the extent of the deep optical observations; Natali et al. 1992). For NGC 628, assuming uniformly-distributed constant star formation over a Hubble time leads to an expected stellar outer disk mass of $5 \times 10^{8} M_{\odot}\left(0.45 M_{\odot}\right.$ $\mathrm{pc}^{-2}$ ), well within our measured mass of $8.4 \times 10^{9} M_{\odot}$ and within the stellar mass inferred from the measured surface brightness $\left(M_{\star}<1.4 \times 10^{9} M_{\odot}\right.$ for a population with $\Upsilon<3$ ). We conclude that the outer disk may have been forming stars for the entire lifetime of the galaxy at approximately the current star formation rate.

\subsection{Similarities between the young and old components}

We now compare the outer disk velocity dispersions of the young $(<10 \mathrm{Myr})$ and old (100 Myr - 10 Gyr) populations, traced by the $\mathrm{H} \alpha$ knots and $\mathrm{PNe}$ (Herrmann et al. 2009), respectively, and find little difference $\left(\sim 11 \mathrm{~km} \mathrm{~s}^{-1}\right.$ vs. $\sim 12 \mathrm{~km} \mathrm{~s}^{-1}$; a small difference may be allowed if we treat the $\mathrm{H} \alpha$ velocity dispersion as a true upper limit, however). Because the difference is unlikely to be much larger than the $1 \sigma$ error of the measurement, we cannot say that there is a significant difference in the kinematic or structural properties between the young and old outer disk populations.

A powerful tracer of the evolution of stellar populations of different ages is the increase of velocity dispersion with age, or, alternatively, the varying asymmetric drift of various populations. The observed low $\sigma_{z}$ of the $\mathrm{H} \alpha$ knots immediately suggests that the degree of asymmetric drift will be small. If one considers that some fraction of the observed value must reflect the intrinsic dispersion of the gas, then the asymmetric drift will be even smaller. The other population that has been measured, PNe, also has a low velocity dispersion and the inferred asymmetric drift is also relatively small $\left(<15 \mathrm{~km} \mathrm{~s}^{-1}\right.$; Herrmann et al. 2009). With the current measurements we cannot conclude that there has been any increase in the velocity dispersions between the very young $\mathrm{H} \alpha$ knots and the older PNe (we comment further on this issue in the following section). It is evident that such an investigation will require uncertainties in the velocity dispersions better than $\sim 1 \mathrm{~km} \mathrm{~s}^{-1}$.

\section{SUMMARY AND CONCLUSIONS}

We have measured the kinematics of $\mathrm{H} \alpha$ knots in the outer disk of NGC 628. We find the stellar disk (traced by the $\mathrm{H} \alpha$ knots) to have a low velocity dispersion, suggesting an undisturbed extended stellar disk. NGC 628 differs from the better-known outer disks of M83 and M94, which were likely accentuated by recent disturbances from neighbors, but resembles (both in optical and kinematic profiles) the outer disks detected in other relatively undisturbed nearby edge-on disks by Christlein \& Zaritsky (2008), supporting the idea that outer disk star formation can be a low-level and ongoing phenomenon in isolated galaxies.

We find $\sigma_{z}$ of the $\mathrm{H} \alpha$ knots to be $<11 \mathrm{~km} \mathrm{~s}^{-1}$ between $1-1.8 R_{25}(13.5-23.2 \mathrm{kpc})$. We adopt a scaleheight similar to the known flaring gas profiles of outer disks $\left(h_{z}=700 \mathrm{pc}\right)$ and estimate a mass density $\Sigma=7.5$ $\mathrm{M}_{\odot} \mathrm{pc}^{-2}$ that can be entirely explained by the observed gas and stars in the outer disk. If the $\mathrm{H} \alpha$-hosting disk is actually much thinner than the flaring gas disk, more dark matter in the outer regions would be allowed. Assuming that outer disk star formation has been going for a Hubble time does not violate either the surface brightness nor surface mass constraints. The high incidence of outer disks (c.f. Christlein \& Zaritsky 2008) suggests that the star formation is not a rare phenomenon - here we show that current limits cannot exclude long-lived outer disk star formation.

Finally, the velocity dispersion of $\mathrm{PNe}$ towards the outer disk of NGC 628 (Herrmann et al. 2009) is nearly the same as that of the $\mathrm{H} \alpha$ knots (the discrepancy grows slightly when considering the dispersion of the $\mathrm{H} \alpha$ knots as a strict upper limit, although any plausible difference remains small). This can result either if the $\mathrm{PN}$ population is rather young (so that scattering has not had a chance to enlarge the dispersion significantly), if there is very little scattering, or if scattering occurs primarily in a single (or few) discrete events that occurred prior to the creation of the bulk of the PNe. We do not expect the outer disk to be exclusive young (see above) and we do expect some level of scattering in outer disks (if not from the classical spiral arms and molecular clouds of inner disks, then perhaps from satellites and dark halo substructure). The solution may be found in the Kazantzidis et al. (2009) simulations, which show that outer disk heating is dominated by the most massive infall event of halo substructure onto the disk, so that stellar populations of different ages do not necessarily have different velocity dispersions. A larger sample of outer disk kinematic measurements could be used to constrain the rate and impact of such infall events.

\section{ACKNOWLEDGMENTS}

This work made use of THINGS, 'The H I Nearby Galaxy Survey' (Walter et al. 2008). DZ and SHF were supported by NSF AST-0907771 and NASA LTSA NNG05GE82G. SJK was supported by NSF AST0401547. 


\section{REFERENCES}

Bell, E.F. \& de Jong, R.S. 2001, ApJ, 550, 212

Bessell, M.S., Freeman, K.C., Wood, P.R. 1986, ApJ, 310, 710

Bush, S.J., Cox, T.J., Hernquist, L., Thilker, D., Younger, J.D. 2008, ApJL, 683, 13

Christlein, D., Zaritsky, D. 2008, ApJ, 680, 1053

Corbelli, E., Salpeter, E.E. 1993, ApJ, 419, 104

Dressler, A., Hare, T., Bigelow, B.C., Osip, D.J. 2006, SPIE, 6269,13

de Vaucouleurs, G., de Vaucouleurs, A., \& Corwin, H.G. 1976, Second Reference Catalogue of Bright Galaxies (Austin : Univ. of Texas Press)

Fathi, K., Beckman, J.E., Zurita, A., Relaño, M., Knapen, J. H., Daigle, O., Hernandez, O., Carignan, C. 2007, A\&A, 466, 905

Ferguson, A.M.N., Wyse, R.F.G., Gallagher, J.S., Hunter, D.A. 1998, ApJ, 506, 19

Gil de Paz, A. et al. 2005, ApJL, 627, 29

Gil de Paz, A. et al. 2007, ApJS, 173, 185

Herbert-Fort, S. et al. 2009, ApJ, 700, 1977

Herrmann, K.A., Ciardullo, R., Feldmeier, J.J., Vinciguerra, M. 2008, ApJ, 683, 630

Herrmann, K.A., Ciardullo, R. 2009, ApJ, in press

Kazantzidis, S., Zentner, A.R., Kravtsov, A.V., Bullock, J.S., Debattista, V.P. 2009, ApJ, 700, 1896

Kamphuis, J. \& Briggs, F. 1992, A\&A, 253, 335
Larson, R.B., Tinsley, B.M. 1978, ApJ, 219, 46

Martin, D.C. et al. 2005, ApJ, 619, 1L

Merrifield, M.R. 1992, AJ, 103, 1552

Moffett, A.J., Kannappan, S.J., Laine, S., Wei, L.H., Baker, A.J., Impey, C.D. 2009, arXiv0908.4232

Natali, G., Pedichini, F., Righini, M. 1992, A\&A, 256, 79

Olling, R.P. 1996, AJ, 112, 457

Pflamm-Altenburg, J., Kroupa, P. 2008, Nature, 455, 641

Roškar, R., Debattista, V.P., Stinson, G.S., Quinn, T.R.,

Kaufmann, T., Wadsley, J. 2008, ApJL, 675, 65

Roškar, R., Debattista, V.P., Quinn, T.R., Stinson, G.S.,

Wadsley, J. 2008, ApJL, 684, 79

Thilker, D.A. et al. 2005, ApJL, 619, 79

Thilker, D.A. et al. 2007, ApJS, 173, 538

Toomre, A. 1964, ApJ, 139, 1217

Toth, G., Ostriker, J.P. 1992, ApJ, 389, 5

Trujillo, I., Martinez-Valpuesta, I., Martínez-Delgado, D.,

Peñarrubia, J., Gabany, R.J., Pohlen, M. 2009, ApJ, 704, 618 van der Kruit, P.C. 1988, A\&A, 192, 117

Walter, F., Brinks, E., de Blok, W.J.G., Bigiel, F., Kennicutt,

R.C., Thornley, M.D., Leroy, A. 2008, AJ, 136, 2563

Zaritsky, D., Christlein, D. 2007, AJ, 134, 135 